\title{
Autoren dieses Bandes
}

1. Univ.-Prof. Dr. Achim Arbeiter

Lehrstuhl für Christliche Archäologie und Byzantinische Kunstgeschichte

Georg-August-Universität Göttingen

Nikolausberger Weg 15

37073 Göttingen

aarbeit@gwdg.de

2. Prof. Dr. Alexander Beihammer

University of Cyprus

Department of History and Archeology

Apostolides Building

Kallipolleos 10

CY-1055 Nicosia

Zypern / Cyprus

abeihamm@ucy.ac.cy

3. Prof. Dr. Wolfram Brandes

Max-Planck-Institut für Europäische Rechtsgeschichte

Hausener Weg 120

60489 Frankfurt a. M.

brandes@mpier.uni-frankfurt.de

4. Daniel Föller

Johannes Gutenberg-Universität Mainz

Fachbereich 07: Geschichts- und Kulturwissenschaften

Historisches Seminar, Abt. V: Byzantinistik

Jakob-Welder-Weg 18

55099 Mainz

foeller@uni-mainz.de

5. Prof. Dr. Johannes Fried

c/o Johann Wolfgang Goethe-Universität Frankfurt

Historisches Seminar

Lehrstuhl für Mittelalterliche Geschichte I

Grüneburgplatz 1

60629 Frankfurt a. M.

fried@em.uni-frankfurt.de 
6. Prof. Dr. Johannes Heil

Erster Prorektor

Hochschule für Jüdische Studien in Heidelberg

Friedrichstraße 9

69117 Heidelberg

johannes.heil@hfjs.uni-heidelberg.de

7. PD Dr. Kay Peter Jankrift

Elias-Holl-Str.11

86391 Stadtbergen

iska.jankrift@t-online.de

8. Univ.-Prof. Dr. Nikolas Jaspert

Ruhr-Universität Bochum

Lehrstuhl für die Geschichte des Späten Mittelalters

Universitätsstraße 150

Gebäude GA 4/31

44801 Bochum

nikolas.jaspert@ruhr-uni-bochum.de

9. Prof. Dr. Bianca Kuehnel

Director of the European Forum at the Hebrew University and Jack-Cotton-Professor of Architecture and Fine Arts, Department History of Art

The Institute of Arts and Letters, Faculty of Humanities

Hebrew University of Jerusalem

Mt. Scopus Campus

IL - 91905 JERUSALEM

ISRAEL

kuehnel@mscc.huji.ac.il

10. Dr. Bettina Krönung

Neckarstraße 8

65462 Ginsheim-Gustavsburg

bettinakroenung@yahoo.de

11. Univ.-Prof. Dr. Johannes Pahlitzsch

Johannes Gutenberg-Universität Mainz

Fachbereich 07: Geschichts- und Kulturwissenschaften

Historisches Seminar, Abt. V: Byzantinistik

Jakob-Welder-Weg 18 
55099 Mainz

[Philosophicum, Raum 03-579]

Tel. 06131-3927190

pahlitzsch@uni-mainz.de

12. PD Dr. Thomas Pratsch

c/o Prosopographie der mittelbyz. Zeit

Berlin-Brandenburgische Akademie d. Wiss.

Jägerstraße 22/23

10117 Berlin

pratsch@bbaw.de

13. Karl Schmitt-Korte

Von-Behring-Str. 142

63075 Offenbach

Tel./Fax: 069-843342

14. Prof. Dr. Wout Jac van Bekkum

Rijksuniversiteit Groningen

Faculty of Arts

Dep.: Semitic Languages and Cultures

Boteringestraat 23

NL - 9712 GC GRONINGEN

NIEDERLANDE

w.j.van.bekkum@rug.nl

W.J.van.Bekkum@rug.nl

15. Prof. Dr. Annette Weber

Hochschule für Jüdische Studien in Heidelberg

Friedrichstraße 9

69117 Heidelberg

annette.weber@hfjs.eu

16. Univ.-Prof. Dr. Dorothea Weltecke

Professur „Geschichte der Religionen und des Religiösen in Europa“

Geisteswissenschaftliche Sektion

Fachbereich Geschichte und Soziologie

Universität Konstanz

Fach 12

78457 Konstanz

Dorothea.Weltecke@uni-konstanz.de 
